\title{
ERROR QUANTIFICATION TOOL OF HEALTH RECORDS FOR DEVELOPING COUNTRIES
}

\author{
Rose Nakasi $^{1}$, Ernest Mwebaze ${ }^{2}$, Aminah Zawedde ${ }^{1}$ and Gilbert Maiga ${ }^{1}$ \\ ${ }^{1}$ Makerere University, Uganda \\ ${ }^{2}$ Google AI
}

\begin{abstract}
In developing countries, the greatest information sources for disease prevalence are official Electronic Health Records (EHR). However, data is usually affected by human error which flaws decisions by stakeholders. The previous reviews considered data quality findings at only an aggregated level, however, this method is vulnerable to errors if wrong data is aggregated and therefore hard to trace the error. This exploratory study though limited, utilized a statistical approach for computing differences between paper and EHR records. A sample of selected health facilities was used as a base study and results indicated varying errors at each level of reporting tool.
\end{abstract}

\section{KEYWORDS}

Paper Health Records, Online Health Records, Error Quantification

\section{INTRODUCTION}

Electronic Health Records provide statistics about disease distribution cases which can adjust accuracies of disease modeling in a certain location at a given period of time. Therefore, for stakeholders to rely on such data, it must be reliable which isn't the case now (WHO, 2O11) and the fact that EHR is aggregated data, it is hard to track the errors from the source.

Taking a Case for Uganda, a developing country, the National eHMIS (electronic Health Management Information system) aggregates diagnosis cases monthly from health facilities that are in the system. The records are captured at health facilities daily and aggregated to weekly and then sent to the District Health officers who electronically capture these records into the system using data entrants. We believe this kind of system is prone to errors and must be investigated.

Error quantification in health records can contribute to a reliable source of health record data. It's even more useful if one wants to use the eHMIS data but has no idea of the error to account for in their analysis. The Uganda Ministry of Health $(\mathrm{MOH})$ recognizes that improper data quality checks in the eHMIS system, unrealistic analysis using such data will affect true representation of the output from the analysis. Crucial in dealing with limitations in eHMIS, error quantification process must start from the health facility where the records are captured from (the laboratory) and then monitor captured and aggregated records on weekly and monthly basis both at facility and district level. In this research, we believe that proper tracking of daily records at health facility level provides a more realistic audit track and trail of the health records before and after they are aggregated in the weekly and monthly records.

Error quantification will undoubtedly increase the level of confidence to be placed on health data for effective disease distribution modeling. Here, we describe a simple statistical method based on the difference measure that makes a comparison of records at each reporting level implemented through a developed software tool that quantifies the error in reporting records. This method is utilized from the source of data at the health facility and spans through at the aggregated level at district level. We further incorporate the analysis tool into a web based platform that reliably helps to monitor and trace the error with a user interface. 


\section{RELATED WORK}

With the increasing integration of electronic health records in health care, there is a growing interest in using such data sources to enhance the disease distribution modeling. Literature acknowledges the use of multiple data sources in modeling (Moore et al., 2016). The use of health records for disease distribution modeling is also gaining momentum in literature for example (Xia et al, 2013) showed how using Electronic Health Records (EHR) can improve modeling disease severity in multiple sclerosis, it's also demonstrated in another study using a Gaussian process framework to show how Health Management Information system data helps in mapping malaria across a changing territory delimitation in Uganda (Andrade-Pacheco, Mubangizi \& Quinn., 2014). In this study however, the authors emphasize improving quality of data from the eHMIS if realistic response from decision makers is to be achieved.

\subsection{Error Quantification}

Recently, different studies (Stevenand et al., 2016) and (Jones et al., 2015) drew attention on citing biases associated with disease patterns found in EHR and associated complications arising if such data is used to calculate disease prevalence. In response, research efforts have gone into understanding the pattern of bias in the medical records. A 2017 study was conducted comparing paper based and electronic records without knowledge of the data in the electronic abstracts (Altrock et al., 2017). General coding rules were followed. Samples were picked from a government hospital and the study was conducted in 2001 for four months. Quantitative parameters were reported with absolute and relative frequencies, and distribution characteristics were reported using mean, median, and range.

World Health Organization has also carried out research on data quality assessment of health records in different countries including Uganda (WHO, 2011). For the 2010-2011 report, analyses were made on the district monthly reporting, missing and zero values and a computation of a percentage difference between monthly and end of year data at national level. A comparison among district reports was conducted and districts with poor data quality listed. A similar report by WHO has been conducted in Cambodia for 2012 to compare monthly paper records with the electronic results. It was found that Cambodia has a well-functioning system with a major recommendation of conducting facility data verification (WHO, 2012). While these studies provide impressive insights to error quantification, there is still lack for a systematic way to trace the reporting errors from source. Additionally, a software tool to quantify the errors is an added advantage to allow stakeholders easily and quickly account for the errors in eHMIS.

\section{DATA AND METHODS}

This research assessed the veracity of records by adopting statistical analysis of the raw records from daily, weekly and monthly records and the electronic records from weekly and monthly aggregated records to quantify the error rate. Comparison was made and presence of error reported as illustrated in Figure 1.

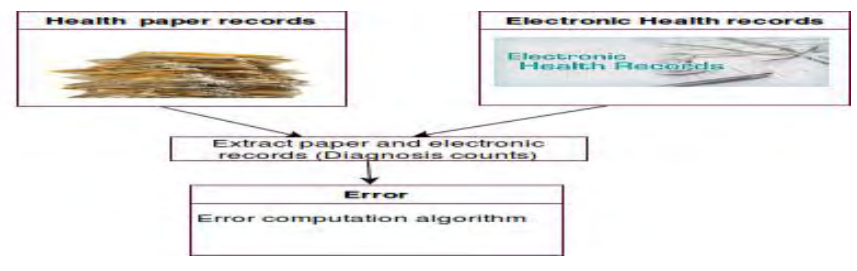

Figure 1. Methodology flow for error quantification

\subsection{Data}

1) Data variables to be extracted: Health records in a developing country like Uganda were captured at three levels, namely; 
a) On a daily basis; these were captured in register books at the health facility. From these records, the study captured demographic data variables (date of diagnosis, name, age, gender) and clinical variables (disease, method of diagnosis, result of diagnosis). The patient level information like date of diagnosis, name, disease diagnosed, and diagnosis cases. Noteworthy is that this information is not captured in the eHMIS.

b) Weekly; these were aggregated records on a weekly basis from health facilities on paper and later taken to the district health centers for aggregation.

c) Monthly; these were aggregated from the weekly records and captured before recording at the district level. Here, we extracted only information on the disease under investigation.

2) Data extraction approach: From the facilities, scanned copies and records were manually extracted and tabulated in a csv file, uploaded into the health analysis tool and further analysis performed. Additionally, records in eHMIS, MOH provided data about the aggregated malaria cases from health facilities under study.

\subsection{Analysis}

Statistical methods; assessing the variability of the samples, a statistical quantitative parameter of difference was used to compare any two samples. Results were calculated using csv file.

difference $=X_{i}-Y_{J}$

where $X_{i}=X_{1}, X_{2}, \ldots X_{n}$ and $Y_{1}, Y_{2}, \ldots Y_{n}$.

The difference was computed over a given period of time and spatial environment and a comparison between the results in the raw paper records and the eHMIS computed and quantified.

The error difference rates isn't generalized across all districts but a similar approach can be adopted across similar tasks. Finally, we should weigh the magnitude of the error in different localities with in the country from the specified health facilities under study and indicate the rate of error in each.

\subsection{System Development for Health Data Analysis Tool}

The tool developed in the study (see interface in Figure 2) was designed to upload scanned paper and electronic health records at health facilities for all levels. The records were tabulated into excel or csv sheet and then analysis was performed on data with guidance of a user interface that was developed using PHP and JavaScript programming languages and can run on any web browser. It further provided visualization for the spatial magnitude of the error on the map using geo coordinates of the different health facilities on a google map.

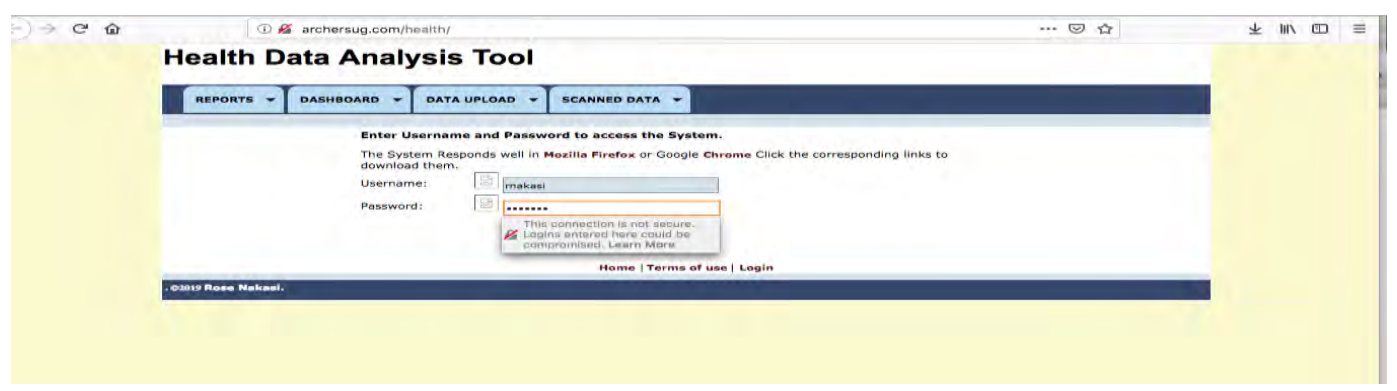

Figure 2. Health data analysis tool to visualise error magnitude between paper and electronic based records 


\section{RESULTS}

A web based health data analysis tool was developed which quantifies error between diagnosis records of paper based and electronic health results. The tool facilitates paper based records at health facilities that have been scanned to be uploaded daily, weekly and monthly basis and equally electronic health records at district level and facility level on monthly basis. Using statistical measure of the difference, a comparison was done among the daily, weekly (paper based) and monthly records (from the eHMIS data).

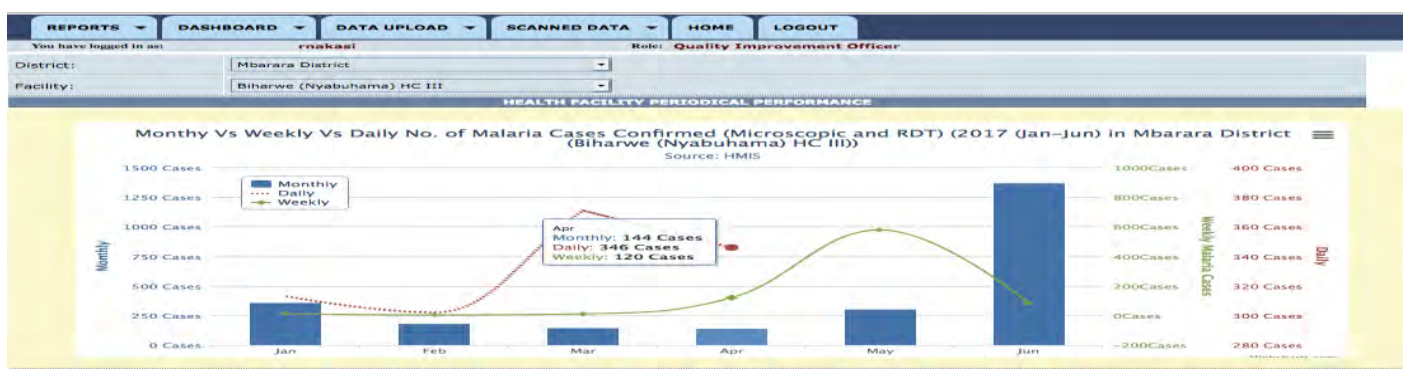

Figure 3. Monthly Vs Weekly Vs Daily number of malaria cases confirmed malaria positive in Biharwe HCIII

Sampling on Biharwe HCIII, a visualisation graph showed the records difference from the system between January and June 2017 (see Figure 3). From the graph, the results show a huge record difference from each of the datasets that is scanned paper records for aggregated daily per month, aggregated weekly per month and monthly (from the eHMIS data) from January to June 2017. Using the month of April, there were huge disparities in the number of malaria diagnosed cases for each dataset which indicate 346 daily paper records, 120 weekly paper records and 144 monthly eHMIS records.

The analysis tool also presented a difference between the health records for all the three health facilities in Mbarara district on a satellite map of Uganda using geo coordinates of facilities through Google maps. Sampling Biharwe HCIII indicates difference between records as visualised on the map in Figure 4 and the tabular representation of the same in Table 1.

Table 1. eHMIS monthly and scanned paper records of Biharwe per month from January to June 2017

\begin{tabular}{lllllll}
\hline Record & Jan & Feb & Mar & April & May & June \\
\hline eHMIS & 135 & 73 & 60 & 53 & 117 & 805 \\
Paper & 123 & 73 & 0 & 53 & 0 & 805 \\
Difference & -12 & 0 & -60 & 0 & -117 & 0 \\
\hline
\end{tabular}

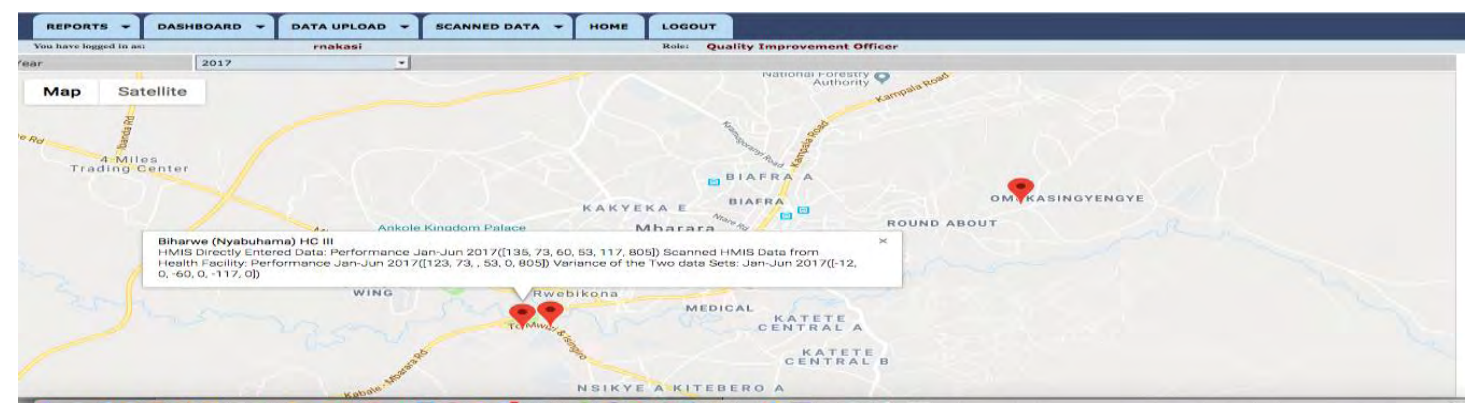

Figure 4. Spatial Visualisation of the difference at different health facilities 


\section{CONCLUSION AND FUTURE WORK}

By analysing the results from this study, our approach retains the advantage of realistically analysing and quantifying the reporting error in health records which would provide accurate results to stakeholders. Additionally, the web based health data analysis tool acts a basic prototype that can be adopted in similar tasks with a lot of ease and act as a basis tool for designing new automated Electronic health systems. In the study the error does not only happen with aggregated data but out of a ripple effect from the source and through all levels of reporting as well. In future, semantic automation and analysis from a big spatial environment that covers a bigger part of the country should be conducted for a more accurate generalisation of the error margin. After collecting enough data for analysis, the web based reporting tool could be improved further to provide an error prediction mechanism. A point of care diagnostic system can also be developed which enables real time diagnosis and feed results directly into the electronic Health Management Information System. This way, manual entry of the health records is eliminated.

\section{ACKNOWLEDGEMENT}

The authors would like to thank Ministry of Health and Health facilities for providing data and authorization to use health records for this study and Swedish International Development Agency (SIDA) for funding this research.

\section{REFERENCES}

Altrock P.M, Ferlic J, Galla T, Tomasson M.H and Michor F, 2017. A computational model of mgus progression to multiple myeloma identifies optimum screening strategies and their effects on mortality." doi: https://doi.org/10.1101/208645.

Andrade-Pacheco R, Mubangizi M and Quinn J, 2014. Consistent mapping of government malaria records across a changing territory delimitation. Malaria Journal.

Jones T.F, Dunn J, Jones S, Marder E and Garman K, 2015. Assessment of administrative claims data for public health reporting of salmonella in Tennessee. J. Am. Med. Inform. Assoc., 22 (e1) (, pp. e34-e38).

Moore A.W, Anderson B, Das K and Wong W.K, 2006. Combining multiple signals for biosurveilance. Ist ed., M. M Wanger, A. W. Moore and R. M Aryel eds. Elservier Inc, 2006, ch 15, pp. 321-331.

World Health Organisation, 2011. Assessment of health facility data quality." Data quality report card Uganda. WHO press, Geneva Tech Rep.

World Health Organisation, 2012. Assessment of health facility data quality. data quality report card cambodia, WHO press, Geneva Tech Rep.

Stevenand C, Bagley B, Russ and Altman B, 2016. Computing disease incidence, prevalence and comorbidity from electronic medical records. Journal of Biomedical Informatics; Volume 63, Pages 108-111.

Xia Z, Secor E, Lori B, Riley M, Bove M, Cheng S, Chitnis T, Cagan A and Vivian S, 2013. Modeling disease severity in multiple sclerosis using electronic health records. Doi: 10.1371. 